EUROPEAN ORGANIZATION FOR NUCLEAR RESEARCH

CERN AT/93-34 (MA)

LHC Note 243

Tracing Back Measured Magnetic Field Imperfections in LHC Magnets by Means of the Inverse Problem Approach

S. Russenschuck, T. Tortschanoff, A. Ijspeert, N. Siegel, R. Perin

\begin{abstract}
After measuring the magnetic field of a model or prototype superconducting magnet for the Large Hadron Collider (LHC) an inverse field problem is formulated in order to explain the origin of the content of unwanted multipole terms. The inverse problem solving is done by means of a least-squares minimization using the Levenberg-Marquard algorithm. Although the uniqueness of the results remains uncertain, useful insights into the causes of measured field imperfections can be deduced. A model dipole magnet, a main quadrupole prototype and a combined dipole-sextupole corrector magnet are given as examples.
\end{abstract}

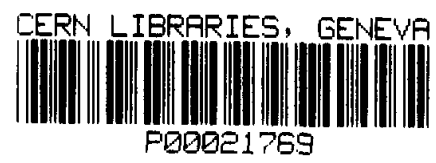

13th International Conference on Magnet Technology (MT13), Victoria, Canada 20-24 September 1993

Geneva, Switzerland $02 / 17 / 94$ 


\title{
Tracing Back Measured Magnetic Field Imperfections in LHC Magnets by Means of the Inverse Problem Approach
}

\author{
S. Russenschuck, T. Tortschanoff, A. Ijspeert, N. Siegel, R. Perin \\ CERN, 1211 Geneva 23, Switzerland
}

\begin{abstract}
After measuring the magnetic field of a model or prototype superconducting magnet for the Large Hadron Collider (LHC) an inverse field problem is formulated in order to explain the origin of the content of unwanted multipole terms. The inverse problem solving is done by means of a least-squares minimization using the Levenberg-Marquard algorithm. Although the uniqueness of the results remains uncertain, useful insights into the causes of measured field imperfections can be deduced. A model dipole magnet, a main quadrupole prototype and a combined dipolesextupole corrector magnet are given as examples.
\end{abstract}

\section{INTRODUCTION}

CERN is preparing for the construction of a new high energy collider, the Large Hadron Collider (LHC). This new facility is based on a double ring of high field superconducting magnets installed in the $26.7 \mathrm{~km}$ long LEP tunnel, above the LEP machine components. Several model and prototype magnets have been built in common development programs with industry and national laboratories [1]. Their measured field distribution exhibits multipole components which are due to perturbations of the coil block arrangements in the manufacturing process. In order to reduce these random errors it is necessary to know where they come from, thus allowing to specify appropriate tolerances for the construction.

The paper discusses the inverse field calculation problem associated with this question.

\section{THE INVERSE FIELD PROBLEM}

The function to be minimized in the inverse field computation problem yields

$$
\min z(\vec{X})=\min \sum_{i=1}^{9} p_{i} \cdot\left(\left(f_{i}(\vec{X})\right)^{2}+\left(g_{i}(\vec{X})\right)^{2}\right)
$$

with the residuals

$$
\begin{aligned}
& f_{i}(\vec{X})=b_{i}^{*}(\vec{X})-b_{i} \\
& g_{i}(\vec{X})=a_{i}^{*}(\vec{X})-a_{i}
\end{aligned}
$$

subject to upper and lower bounds for the design variables $x_{l l} \leq x_{l} \leq x_{i u}, l=1, . . n$ where $b_{i}^{*}(\vec{X}), a_{i}^{*}(\vec{X})$ are the calculated and $b_{i}, a_{i}$ are the measured multipoles. $\vec{X}$ is the vector of the design variables for the inverse problem. The $p_{i}$ are weighting factors in order to compensate for the different numerical values of the residuals.

The design variables are the possible perturbations of the coil blocks, which determine the content of the multipole components. The perturbations are due to tolerances in the manufacturing of the conductors, the insulation, the coil blocks and the wedges. In addition positioning errors may occur due to the outward electromagnetic forces and the initial prestress that must be applied to avoid tensile stress over the whole cross section. Because of the non-symmetric nature of the geometrical coil positioning errors a high number of design variables results for the inverse field problem. It had therefore to be assumed that the positioning errors hold for an entire coil block rather than individual conductors. The multipole content of the field in the aperture is evaluated by means of the computer program ROXIE [2] which, starting from the initial design, calculates the positions of the blocks in the deformed coil and calculates the magnetic field, using Biot-Savart law, from line currents at strand location.

The optimization procedure consists of an algorithm for finding the minimum value of an unconstrained objective function as there are no nonlinear constraints to be considered as is often the case in design optimization. The treatment of upper and lower bounds for the perturbations (design variables) is identical to the method used in [3] and which is derived from an exterior point penalty function method. As minimization algorithm the LevenbergMarquard [4] method has been applied which was originally developed for nonlinear regression problems using least squares objective functions. We can apply it therefore to our inverse field problem. Assuming the objective function given by

$$
\min z(\vec{X})=\min \vec{F}(\vec{X})^{T} \vec{F}(\vec{X})
$$

with the residues $f_{i}$ arranged in the vector

$$
\vec{F}(\vec{X})=\left(f_{1}(\vec{X}), f_{2}(\vec{X}), \ldots ., f_{k}(\vec{X})\right)
$$

and the Jacobi Matrix $J(\vec{X})$ of $z(\vec{X})$ it yields:

$$
\nabla z(\vec{X})=2 \cdot J(\vec{X})^{T} \vec{F}(\vec{X})
$$




$$
\nabla^{2} z(\vec{X})=2 \cdot J(\vec{X})^{T} J(\vec{X})+2 \frac{\partial J(\vec{X})}{\partial \vec{X}} \vec{F}(\vec{X})
$$

Using an quadratic approximation of $z(\vec{X})$ and neglecting the second term in eq. 7 the stepsize and direction is given:

$$
\Delta \vec{X}=-\frac{1}{2}\left[J(\vec{X})^{T} J(\vec{X})+\lambda I\right]^{-1} \cdot J(\vec{X})^{T} \vec{F}(\vec{X})
$$

$\lambda I$ can be regarded as an approximation for the neglected term. With a high $\lambda$ the algorithm starts in a GaussNewton direction and $\lambda$ is decreased in the optimization procedure because the neglected term gets less and less important with smaller and smaller residuals.

\section{THE SUPERCONDUCTING DIPOLE MAGNET}

The superconducting model magnet built by JeumontSchneider has been described in detail in [5]. It is a two-inone design, the inside diameter of each coil is $50 \mathrm{~mm}$ and the axes of the two coils are separated by $180 \mathrm{~mm}$. Each coil is formed of two shells of cables arranged in blocks, surrounded by two-in-one aluminium alloy collars. The structure is surrounded by an iron yoke which provides a return path for the flux. The cables are Rutherford type trapezoidal cables made of $\mathrm{NbTi} / \mathrm{Cu}$ composite strands. The coil block arrangement is supposed to be symmetric with respect to the poles. The magnet is operated at a field of 0.6 Tesla at injection and can reach a maximum field of about 10 Tesla. Table 1 . shows the multipole content measured in the center of bore M2 at a radius of $23 \mathrm{~mm}[6]$. The multipoles given are those measured at about $4.8 \mathrm{~T}$ dipole field, where no iron saturation occurs in the yoke and the influence of persistent currents can be neglected. The second column shows the "intrinsic" terms as expected from the ideal coil block arrangement.

\begin{tabular}{|c|c|c|c|c|}
\hline & \multicolumn{2}{|c|}{ Measured } & \multicolumn{2}{c|}{ Intrinsic } \\
\hline $\mathrm{n}$ & Normal & Skew & Normal & Skew \\
\hline 1 & 48534 & - & 48534 & - \\
\hline 2 & -12.04 & -15.04 & - & - \\
\hline 3 & 72.70 & -12.23 & 8.787 & - \\
\hline 4 & -8.39 & -1.94 & - & - \\
\hline 5 & 5.82 & -2.18 & 17.88 & - \\
\hline 6 & -3.06 & 9.66 & - & - \\
\hline 7 & 113.03 & -47.71 & 110.2 & - \\
\hline 8 & -0.78 & 0.83 & - & - \\
\hline 9 & 73.82 & -45.43 & 72.21 & - \\
\hline
\end{tabular}

Table 1: Measured and intrinsic multipole content of the main dipole model magnet in $10^{-4} \mathrm{~T}$ at radius $23 \mathrm{~mm}$, current $7000 \mathrm{~A}$.

The design variables for the minimization problem are the azimuthal and radial displacements of each coil block

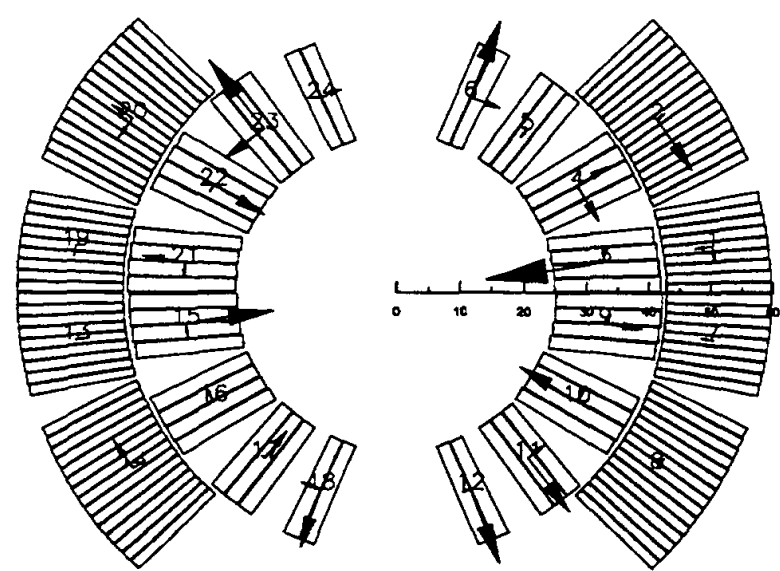

Figure 1: Coil block displacement of the main dipole

thus resulting in 48 design variables. In addition the position of the measurement coil is regarded as a design variable. Figure 1 shows the displacements of the coil blocks found by the Levenberg-Marquard algorithm after about 1200 function evaluations. Because there are far more unknowns than residuals we cannot expect unique solutions to the problem. The algorithm has therefore been started with different initial guesses in order to confirm the solutions, but the differences between these solutions differ only slightly. The arrow in block no. 3 represents a radial displacement of $0.19 \mathrm{~mm}$, all other displacements are to scale.

\section{THE MAIN QUADRUPOLE MAGNET}

The lattice quadrupoles are developed in close collaboration between CERN and CEA, Saclay, in France. The present collaboration agreement foresees design and manufacture of two quadrupole prototypes by CEA, Saclay together with the development of the tooling for a later series production. The main parameters of these magnets which feature, as the dipoles, two apertures in one common yoke, are a nominal gradient of $252 \mathrm{~T} / \mathrm{m}$, a magnetic length of $3.05 \mathrm{~m}$, a nominal current of $15060 \mathrm{~A}$, an inner coil aperture of $50 \mathrm{~mm}$ and an operational temperature of $1.8 \mathrm{~K}$. A design report has been published in [7]. At the time of writing this paper one magnet has been completed and installed in its horizontal test cryostat where it will be subject to power testing and magnetic measurements. Before being assembled into their common yoke the two coil-collar assemblies of the second magnet have undergone magnetic measurements at room temperature. Table 2 gives the measured multipole distribution in the 
straight part of one of these assemblies [8] together with the expected (intrinsic) values.

\begin{tabular}{|c|c|c|c|c|}
\hline & \multicolumn{2}{|c|}{ Measured } & \multicolumn{2}{c|}{ Intrinsic } \\
\hline $\mathrm{n}$ & Normal & Skew & Normal & Skew \\
\hline 1 & - & - & - & - \\
\hline 2 & -21910. & - & -21910. & - \\
\hline 3 & 1.972 & -5.456 & - & - \\
\hline 4 & -0.723 & -0.920 & - & - \\
\hline 5 & -0.219 & -0.436 & - & - \\
\hline 6 & -0.066 & 0.153 & -0.79 & - \\
\hline 7 & -0.004 & -0.285 & - & - \\
\hline 8 & -0.002 & 0.109 & - & - \\
\hline 9 & -0.043 & -0.022 & - & - \\
\hline 10 & 0.59 & -0.044 & 0.52 & - \\
\hline
\end{tabular}

Table 2: Measured multipoles in the straight part of one of the coil collar assemblies and intrinsic values as expected from the coil design in units of $10^{-7} T$ at $15 \mathrm{~mm}$, current $9.3 \mathrm{~A}$.

For the computations certain constraints on the block displacements were introduced: At the poles the collar inserts represent a limitation to any azimuthal motion. The blocks adjacent to the horizontal or vertical planes move together azimuthally; this motion may be different for the two layers. In this way a difference of elastic moduli in the coil layers is accounted for. Each block is allowed to move in the radial direction resulting in a total number of 32 design variables for the inverse field problem. The result of the inverse problem computation can be seen in fig. 2. The arrow length of the most important displacement corresponds to $0.2 \mathrm{~mm}$ in block no. 13. All other displacement arrows are to scale. The arrow in the midplane of the two adjacent coil blocks, e.g. 7 and 10 indicates the direction in which one coil expands respectively the adjacent one compresses. These results also allow the necessity of a mandrel inside the coil aperture in the final collaring phase to be checked (in fact, the coil assembly mandrel was extracted before the final compression of the collars). The displacements in fig. 2 show no significant inside movement of inner layer blocks, indicating that the adopted collaring procedure seems acceptable.

\section{THE COMBINED SEXTUPOLE-DIPOLE CORRECTOR MAGNET}

Each cell in the LHC lattice contains in addition to the dipole bending magnets and the quadrupoles a correction magnet. This magnet incorporates a sextupole coil for correction of the chromaticity of the machine and a dipole coil for correction of the orbit of the particles in the machine. To make the magnet as compact as possible, the dipole coil has been placed around the sextupole coil reducing

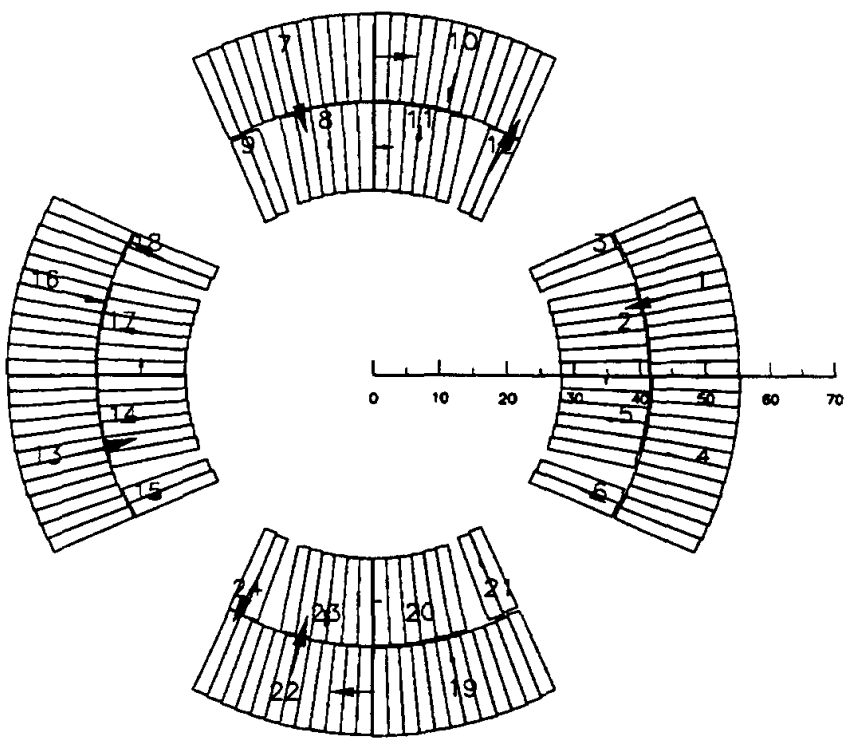

Figure 2: Coil block displacement of the main quadrupole

the overall length of the magnet to $1.3 \mathrm{~m}$. A prototype has been built and tested [9] to see if the concept of superimposed coils (and fields) would give the expected performance. The results showed that, after some training, the magnet could produce all combinations of sextupole fields and dipole fields up to the desired sextupole gradient of $4000 \mathrm{~T} / \mathrm{m} 2$ and dipole field of $1.5 \mathrm{~T}$. It appeared however that the field quality was not as good as one could expect from the calculations, cf. table 3 .

Deformation due to the Lorentz force loading does not seem to be the cause: The measured field precisions are about the same whether measured on the individual fields or on the combined fields where the Lorentz forces are much higher. The measuring coil could not be centered very precisely and this might cause some of the lower multipoles in the sextupole field. In particular the dipole as well as the quadrupole component in the sextupole field can each come from a badly centered measuring coil but not both at the same time because each corresponds to a different centering error. The horizontal dipole field should have no vertical "normal" component. However, the appearance of this component in the measured results can be explained by a known error in the azimuthal position of one of the measuring coils. The precision of the magnet coil assembly was expected to be within $0.05 \mathrm{~mm}$ and this could not explain the measured multipoles.

The inverse problem approach has been applied to see which coil positions would correspond to the measured field quality. All blocks were free to move in the azimuthal and the radial directions resulting in a total number of 56 design variables for the inverse problem. The problem has 
been solved in two steps. The first step was made assuming that the measuring coils were perfectly centered in the magnet. In the second step the position of the measuring coil inside the magnet was also treated as a design variable, increasing the number of unknowns to 58 . In the second step the displacements of the coil blocks found by the algorithm are slightly smaller. The results of the second step are shown in Figure 3 for both the a sextupole and the dipole. The measuring coil was probably off center by $0.038 \mathrm{~mm}$ in the horizontal and $0.031 \mathrm{~mm}$ in the vertical direction. The maximum errors in the block positions of the magnet are a radial displacement of $0.12 \mathrm{~mm}$ of block 1 and a tangential displacement of $0.3 \mathrm{deg}$. in block 7. The measured quadrupole components can be partly explained by the off center of the measurement coil and partly by the inward displacement of blocks 1 to 6 of the sextupole, which also explain the measured octupole. There also seems to be a systematic widening between the sextupole coils.

\begin{tabular}{|c|c|c|c|c|}
\hline & \multicolumn{2}{|c|}{ Measured } & \multicolumn{2}{c|}{ Intrinsic } \\
\hline $\mathrm{n}$ & Normal & Skew & Normal & Skew \\
\hline 1 & - & 10650. & - & 10650. \\
\hline 2 & -70.5 & -58.3 & - & - \\
\hline 3 & 3640. & 4.6 & 3640. & 2.87 \\
\hline 4 & 1.8 & -0.5 & - & - \\
\hline 5 & -0.8 & -2.2 & - & 0.11 \\
\hline 6 & -0.1 & 0.2 & - & - \\
\hline 7 & - & - & - & 0.026 \\
\hline 8 & - & - & - & - \\
\hline 9 & -0.22 & - & 0.074 & -0.03 \\
\hline
\end{tabular}

Table 3: Measured and intrinsic multipole content of the combined sextupole-dipole corrector magnet in $10^{-4} \mathrm{~T}$ at a radius of $10 \mathrm{~mm}$. Current for the sextupole $3200 \mathrm{~A}$ per coil segment, for the dipole $360 \mathrm{~A}$ per coil segment.

\section{CONCLUSIONS}

The inverse problem approach to analyze the measured field quality in the different types of superconducting accelerator magnets has turned out to be a powerful tool to trace back their origin in construction imperfection in a non destructive way. It is an extension of the optimization methods used to design these magnets. Although the higher number of 'design' variables makes the proof for the uniqueness of the results difficult, the computations provide an helpful insight about the possible sources of unwanted multipole components in the magnets. The magnitudes found for the block displacements turned out to be comparable to the mechanical tolerances expected in the magnet coil fabrication. This allows realistic predictions concerning the content of the unwanted multipole components to be expected in LHC.

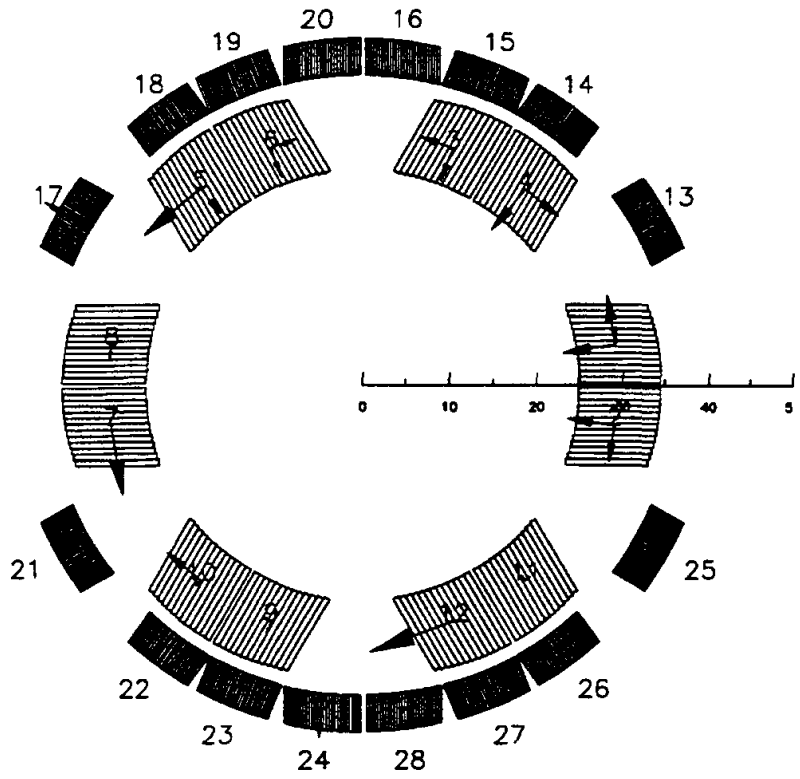

Figure 3: Coil block displacement of the combined dipolesextupole corrector magnet

\section{REFERENCES}

[1] R. Perin: The Superconducting Magnet System for the LHC, IEEE Transactions on Magnetics, Vol 27, No. 2, 1991, pp. 1735-1742.

[2] S. Russenschuck, T. Tortschanoff: Estimation of the Errors of Conductor Positioning in the LHC Main Magnets from a given Multipole Content, IEEE Transactions on Magnetics.

[3] S. Russenschuck: ROXIE, the routine for the optimization of magnet $X$ - sections inverse problem solving and end region design, LHC - note 238, CERN, Geneva 1993

[4] J. Kuester, J.H. Mize: Optimization Techniques with Fortran, McGraw-Hill, 1973.

[5] J.L. Borne, D. Bouichou, D. Leroy, W. Thomi: Manufacturing of High (10 Tesla) Twin Aperture Superconducting Dipole Magnet for L.H.C., IEEE-Transactions on Magnetics, Vol. 28 , No. 1,1992 , pp. $323-326$

[6] L. Walckiers: private communications

[7] J.M. Baze et al, Design and fabrication of the Prototype Superconducting Quadrupole for the CERN LHC project, IEEE- Transactions on Magnetics, Vol 28, No. 1 Jan. 1992, pp 335-337.

[8] J. le Bars, S. Regnaud, Resultats des Mesures Magnetiques a chaud sur Q22, private communication, 18. July 1993.

[9] A. Ijspeert, R. Perin, E. Baynham, P. Clee, R. Coombs, M. Begg, D. Landgrebe: Results of the combined sextupoledipole corrector magnet for LHC, Applied Superconductivity Conference, Chicago, August 1992. 